\title{
GMR
}

\section{Effects of siRNA-mediated silencing of Sal-like 4 expression on proliferation and apoptosis of prostate cancer $\mathrm{C} 4-2$ cells}

\author{
K.F. Liu and Y.X. Shan \\ Department of Urology, The Second Hospital Affiliated to Suzhou University, \\ Suzhou, Jiangsu Province, China \\ Corresponding author: Y.X. Shan \\ E-mail: shanyuxidu@yeah.net \\ Genet. Mol. Res. 15 (2): gmr.15027885 \\ Received October 22, 2015 \\ Accepted December 28, 2015 \\ Published May 13, 2016 \\ DOI http://dx.doi.org/10.4238/gmr.15027885
}

\begin{abstract}
The aim of this study was to evaluate the effects of small interfering RNA (siRNA)-inhibited expression of the Sal-like 4 (SALL4) gene on the proliferation, colony formation, and apoptosis of prostate cancer C4-2 cells. C4-2 cells were cultured and divided into a si-SALL4 group, a negative control siRNA group, and a blank control group. SALL4 mRNA levels and protein expression were detected by real-time polymerase chain reaction and western blot, respectively. Changes in the cell proliferation and colony formation capacities were observed by using the MTS colorimetric method and colony formation assay, respectively. The influence of SALL4 on apoptosis was assessed with flow cytometry, and the expression of apoptosis-related proteins B-cell lymphoma 2 (Bcl-2) and bcl-like-protein 4 (Bax) were detected by western blot. The si-SALL4 group had significantly lower mRNA and protein levels of SALL4 as well as decreased proliferation and colony formation capacities than the negative control group $(\mathrm{P}<0.05)$. There were significantly more apoptotic cells in the si-SALL4 group compared to the negative control $(\mathrm{P}<0.05)$, and the expression of $\mathrm{Bcl}-$ 2 and Bax decreased and increased, respectively, after treatment with
\end{abstract}


si-SALL4. Silencing SALL4 expression by using siRNA technology inhibited the proliferation and colony formation of C4-2 cells, and promoted apoptosis likely mediated by Bcl-2 and Bax expression. These results provide experimental basis for further elucidating the role of SALL4 in prostate cancer cells.

Key words: Small interfering RNA; Sal-like 4; Prostate cancer; Gene silencing

\section{INTRODUCTION}

The mortality rate of prostate cancer ranks second among those of all male malignant tumors worldwide (Jemal et al., 2011). Without obvious early symptoms, many patients with prostate cancer have already experienced metastasis upon diagnosis, resulting in poor treatment outcomes. Therefore, it is necessary to find specific markers for early prediction of the onset and progression of prostate cancer and to develop targeted drugs.

The Sal-like 4 (SALL4) gene was first cloned from the fruit fly in 2006 because it is a widely expressed transcription factor. It encodes proteins with several $\mathrm{C} 2 \mathrm{H} 2$-type zinc finger double domains. In the normal physiological state, SALL4 plays an important role in embryo development and organ formation as an embryonic stem cell factor (Rao et al., 2010). Recently, it has been closely associated with tumor onset and progression. In general, SALL4 is highly expressed in germ cell tumors, gastric cancer, lung cancer, breast cancer, and liver cancer (Cao et al., 2009; Kobayashi et al., 2011; Yong et al., 2013). We have previously reported that SALL4 was highly expressed in prostate cancer tissues and its levels closely correlated with Gleason score, clinical stage, prognosis, and expression of prostate specific antigens. Therefore, this protein may be a crucial marker for the diagnosis and prognostic evaluation of prostate cancer (Lai et al., 2013), and its biological role in the development of this cancer should be further examined. In this study, SALL4 gene expression in the prostate cancer cell line C4-2 was silenced by RNA interference. Cell proliferation and colony formation were detected using the MTS colorimetric assay and colony formation assay, respectively, and apoptosis was detected by flow cytometry. The aim of this study was to assess the effects of the SALL4 protein on the proliferation and apoptosis of prostate cancer cells.

\section{MATERIAL AND METHODS}

\section{Reagents and culture conditions}

RNAiso Plus total RNA extraction kit, reverse transcription kit, polymerase chain reaction (PCR) reagent and Lipofectamine ${ }^{\mathrm{TM}} 2000$ transfection reagent were purchased from Life Technologies (USA). SALL4 rabbit anti-human polyclonal antibody was from Santa-Cruz (USA). Bcl-2 monoclonal antibody was obtained from Abcam (USA). MTS reagent, apoptosisrelated reagent, and serum were provided by Sigma (USA). Incomplete RPMI 1640 medium was purchased from Gibco (USA). All other reagents were from Beyotime Institute of Biotechnology Co., Ltd. (China). Human prostate cancer C4-2 cells were provided by China Center for Type Culture Collection (China). The cells were cultured in RPMI-1640 medium containing 10\% fetal bovine serum and incubated at $37^{\circ} \mathrm{C}$ in $5 \% \mathrm{CO}_{2}$ atmosphere with saturated humidity. 


\section{C4-2 cell transfection}

C4-2 cells were inoculated onto 6-well plates at a density of $2 \times 10^{\%} / \mathrm{m}^{2} 24 \mathrm{~h}$ before transfection, which was initiated at $70-80 \%$ confluence. Then, the cells were divided into an untreated blank control group, a negative control (NC) group treated with NC-small interfering (siRNA), and a si-SALL4 group treated with SALL4 siRNA (RiBo-Bio, Guangzhou, China). Lipofectamine $^{\mathrm{TM}} 2000(5 \mathrm{~mL} / \mathrm{L})$ and different groups of siRNA $(100 \mathrm{pM})$ were dissolved in serum- and antibiotic-free culture medium, mixed, and left at room temperature for $20 \mathrm{~min}$. After the original culture medium was discarded, the cells were washed twice with fresh serum-free culture medium and then the mixture of Lipofectamine ${ }^{\mathrm{TM}} 2000$ and siRNA was added. Culture medium containing serum was used $6 \mathrm{~h}$ later to further culture the cells for $24-72 \mathrm{~h}$.

\section{Detection of SALL4 mRNA levels by real-time PCR}

TotalRNAwasextracted withTRIzolreagent48haftertransfection, andSALL4andinternal reference GAPDH were amplified by using real-time PCR. Primers for SALL4 were: forward 5'-CCGGCAGTAAGGACTGTC-3', and reverse 5'-TCTCTGTCTTTAGGTACACCACA-3' (PCR product: $97 \mathrm{bp}$ ). GAPDH primers: forward 5'-GGAGTCAACGGATTTGGTCGTAT-3', and reverse 5'-AGCCTTCTCCATGGTGGTGAAGAC-3' (PCR product: $500 \mathrm{bp}$ ). PCR was performed at $98^{\circ} \mathrm{C}$ for $20 \mathrm{~s}, 55^{\circ} \mathrm{C}$ for $30 \mathrm{~s}$, and $72^{\circ} \mathrm{C}$ for $30 \mathrm{~s}$ for a total of 32 cycles.

\section{Detection of SALL4, B-cell lymphoma 2 (Bcl-2), and bcl-2-like protein 4 (Bax) protein levels by western blot}

Cells were collected and total protein was quantified $72 \mathrm{~h}$ after transfection, resolved on $8 \%$ SDS-PAGE, and transferred to a PVDF membrane. The membrane was blocked in skim milk at room temperature for $1 \mathrm{~h}$ and incubated overnight with GAPDH $(1: 1000)$, SALL4 (1:1000), Bcl-2 (1:1000) and Bax (1:1000) primary antibodies (SantaCruz, Dallas, TX, USA) at $4{ }^{\circ} \mathrm{C}$. The next day, the membrane was washed with TBS-T, incubated with goat anti-rabbit $\mathrm{IgG} /$ horseradish peroxidase (1:2000 dilution) at room temperature for $1 \mathrm{~h}$, washed again with TBS-T, and treated with ultrasensitive enhanced chemiluminescence reagent.

\section{Detection of cell proliferation by MTS assay}

C4-2 cells were cultured on 96-well plates, and $20 \mu \mathrm{L}$ MTS was added to each well $24,48,72$, or $96 \mathrm{~h}$ after transfection. The cells were then cultured for another $4 \mathrm{~h}$ at $37^{\circ} \mathrm{C}$, and the absorbance was measured at $490 \mathrm{~nm}$. Three replicate were performed for each group and the mean was used as the final result.

\section{Detection of colony formation by colony formation assay}

C4-2 cells were cultured on 6-well plates and those cells adhering to the wall were digested $24 \mathrm{~h}$ after transfection and counted. Two thousand cells from each group were inoculated on 6-well plates and incubated, and the culture medium was discarded 14 days later. The cells were thereafter fixed with $1 \mathrm{~mL}$ paraformaldehyde for $20 \mathrm{~min}$ and stained by adding $1 \mathrm{~mL}$ crystal violet staining solution for $30 \mathrm{~min}$ after removal of paraformaldehyde. 
Subsequently, the cells were washed with pure water, air-dried at $37^{\circ} \mathrm{C}$ and counted under a microscope. A colony was defined where there were more than 50 cells.

\section{Detection of apoptosis by flow cytometry}

Cells $\left(10^{6}\right)$ were collected $72 \mathrm{~h}$ after transfection, washed with PBS, and centrifuged at $1000 \mathrm{~g}$ for $5 \mathrm{~min}$. This procedure was then repeated. After the supernatant was discarded, the cells were collected, mixed, resuspended in $500 \mu \mathrm{L}$ binding buffer, mixed with $10 \mu \mathrm{L}$ propidium iodide (Beyotime, Beijing, China), left at room temperature in the dark for 5-15 min for the reaction to occur, mixed with $5 \mu \mathrm{L}$ AnnexinV-FITC (Beyotime, Beijing, China), and finally detected with flow cytometry. The binding buffer was prepared by Beyotime (Beijing, China).

\section{Statistical analysis}

All data were analyzed by the SPSS19.0 software (Chicago, IL, USA) and are reported as means \pm standard deviation. Inter-group comparisons were performed by using the Student $t$-test or one-way analysis of variance.

\section{RESULTS}

\section{SALL4 siRNA reduced $S A L L 4$ mRNA levels in transfected cells}

Total RNA was extracted $48 \mathrm{~h}$ after transfection and SALL4 gene expression level was detected by real-time PCR (Figure 1A). The relative expression level of SALL4 mRNA of the $\mathrm{NC}$ group was set at $100 \%$ and the si-SALL4 group was then $47.92 \%(\mathrm{P}<0.05)$. As expected, si-SALL4 inhibited SALL4 expression at the mRNA level.

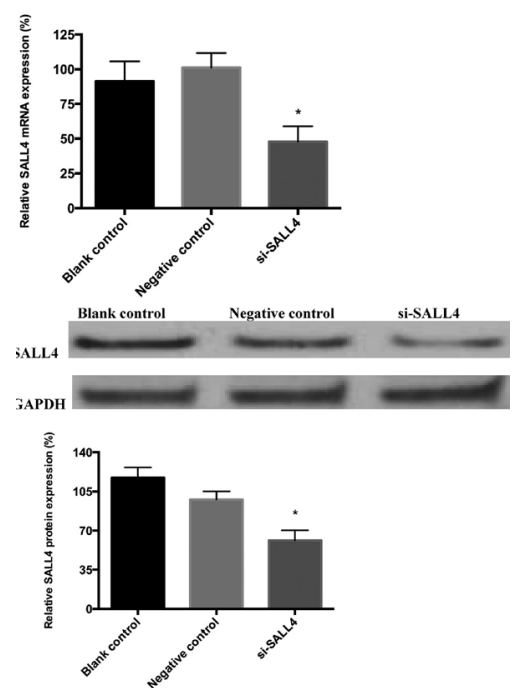

Figure 1. Effects of SALL4 siRNA transfection on SALL4 mRNA levels (top) and protein expression (bottom). A representative western blot for protein expression of SALL4 and GAPDH is shown. All graph values are reported as means $\pm \mathrm{SD}, \mathrm{N}=3, * \mathrm{P}<0.05$ vs negative control. 


\section{SALL4 siRNA inhibited SALL4 protein expression in transfected cells}

Total protein was extracted $72 \mathrm{~h}$ after transfection and detected by western blot (Figure 1B). The expression of SALL4 protein was significantly lower in the si-SALL4 group compared to the control group $(49.71 \%)(\mathrm{P}<0.05)$. Therefore, si-SALL4 also suppressed SALL4 expression at the protein level.

\section{Transfection with SALL4 siRNA inhibited cell proliferation}

Compared with the blank control and $\mathrm{NC}$ groups, the proliferative activity of the siSALL4 group was significantly inhibited $72 \mathrm{~h}$ after transfection $(\mathrm{P}<0.05)$ (Figure 2$)$.

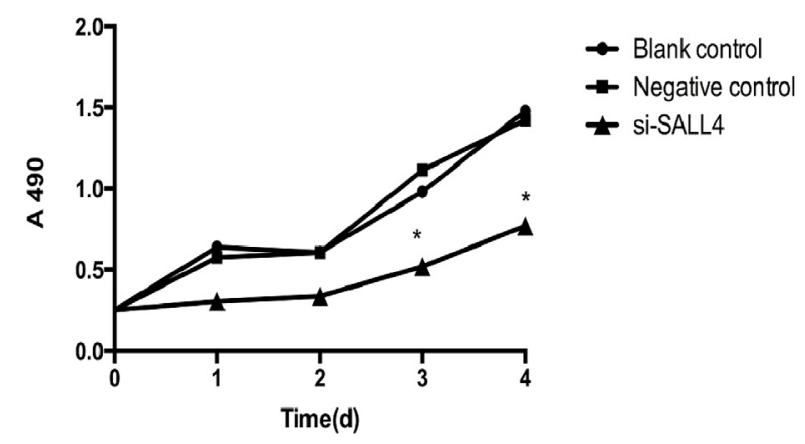

Figure2. Changes in cell proliferationafterSALL4siRNAtransfection. Means $\pm \mathrm{SD}, \mathrm{N}=3,{ }^{*} \mathrm{P}<0.05$ vs negative control.

\section{Transfection with SALL4 siRNA inhibited colony formation}

The colony formation assay was performed $24 \mathrm{~h}$ after transfection and the colony formation rate was detected after 14 days of culture. The si-SALL4 group had significantly fewer colonies than the NC group $(47.72 \%)(\mathrm{P}<0.05)$ (Figure 3$)$.

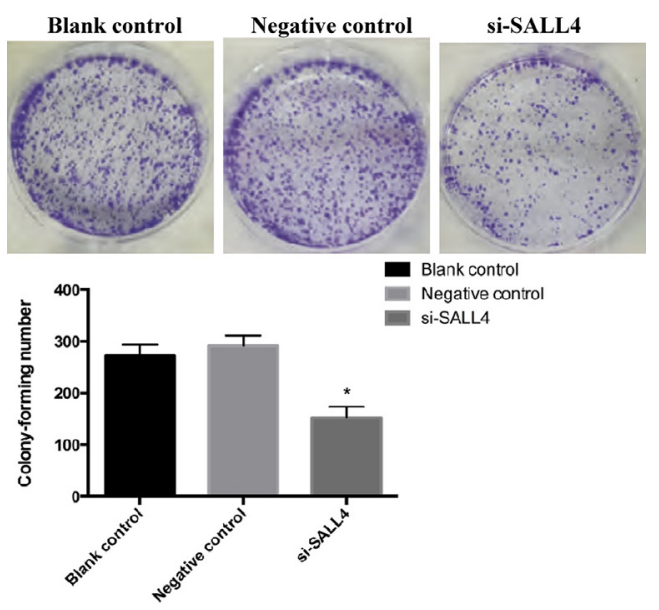

Figure 3. Changes in colony formation after SALL4 siRNA transfection. Representative colonies on plates (top) and graphed values (bottom) are shown. Graph values are reported as means $\pm \mathrm{SD}, \mathrm{N}=3,{ }^{*} \mathrm{P}<0.05$ vs negative control. 


\section{Detection of cell apoptosis by flow cytometry}

The apoptotic rate of the si-SALL4 group was $16.57 \%$, which was significantly higher than the NC group $(\mathrm{P}<0.05)$ (Figure 4).
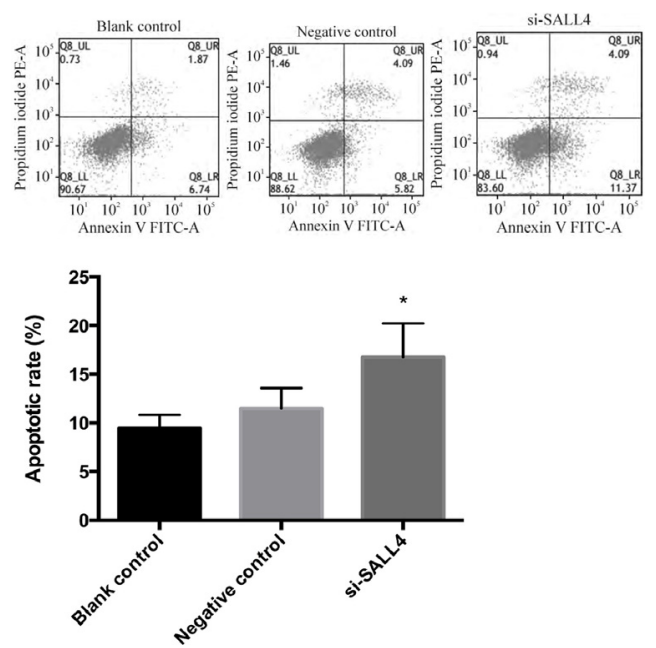

Figure 4. Effects of SALL4 siRNA on cell apoptosis. Results from flow cytometry (top) and graphed values (bottom) are shown. Graph values are reported as means $\pm \mathrm{SD}, \mathrm{N}=3, * \mathrm{P}<0.05$ vs negative control.

\section{Si-SALL4 downregulated Bcl-2 and upregulated Bax protein expression}

Compared with the $\mathrm{NC}$ group, the expression of the anti-apoptotic protein Bcl-2 in the si-SALL4 group was significantly downregulated $(\mathrm{P}<0.05)$, while the expression of the pro-apoptotic protein Bax was significantly upregulated $(\mathrm{P}<0.05)$ (Figure 5$)$.

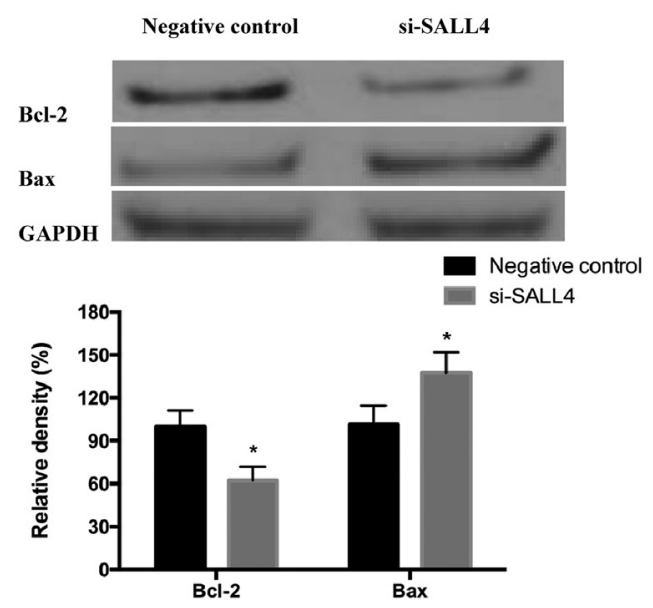

Figure 5. Effects of SALL4 siRNA on Bcl-2 and Bax protein expression in C4-2 cells. Representative western blot (top) and its quantitation (bottom) are shown. Graph values are reported as means $\pm \mathrm{SD}, \mathrm{N}=3, * \mathrm{P}<0.05 v \mathrm{~s}$ negative control. 


\section{DISCUSSION}

Prostate cancer is one of the most devastating malignant tumors in the urogenital system threatening men worldwide and has a rapidly increasing morbidity rate (Liu et al., 2013). However, the pathogenesis of the disease is currently unknown. Malignant tumors, which are typified by uncontrollable proliferation and resistance to apoptosis (Chisholm et al., 2012), are not regulated by defined mechanisms.

The SALL4 gene, an important transcription factor and stem cell factor, plays key roles in the differentiation and development of embryonic organs. Depletion or mutation of SALL4 is associated with diseases such as acro-renal-ocular Okihiro and IVIC syndromes that are characterized by ear deformities, hearing loss, and kidney, heart and limb defects. Highly expressed in many malignant tumors, SALL4 protein may regulate the proliferation and apoptosis of tumor cells, particularly tumor stem cells. SALL4 is commonly overexpressed in malignant tumors. For example, SALL4 is overexpressed in about $90 \%$ of samples of colorectal cancer and its levels closely correlate with lymphatic metastasis and degree of differentiation (Forghanifard et al., 2013). SALL4 expression is increased by $86.1 \%$ in breast cancer samples, even in those at an early stage of disease (Kobayashi et al., 2011). In addition, SALL4 is highly expressed in liver cancer stem cells but not in liver cancer cells; therefore, disrupted expression of SALL4 inhibits proliferation and promotes apoptosis (Oikawa et al., 2013). Yang et al. (2008) reported that SALL4 was crucial to survival and apoptosis in cancer stem cells that were isolated from the promyelocytic leukemia cell line NB4. As shown by gene expression microarray data, inhibiting the SALL4 gene significantly downregulated anti-apoptotic gene Bcl-2 and significantly upregulated pro-apoptotic genes tumor protein 53 and tumor necrosis factor, suggesting that SALL4 maintains the resistance of leukemia cells to apoptosis.

Prostate-specific membrane antigen may positively regulate the growth and migration of prostate cancer cells by upregulating the activity of extracellular signal-related kinase (ERK) protein, and the expression of ERK remarkably resembles that of SALL4 under different conditions. Since phosphorylated ERK may initiate rapid proliferation of tumor cells by activating SALL4 after entering cells, high SALL4 expression may be regulated by prostate-specific membrane antigen in prostate cancer (Brumbaugh et al., 2012). Currently, the regulatory effects of SALL4 on the biological functions of prostate cancer cells have never been reported. Herein, when SALL4 protein expression was inhibited, the proliferation and colony formation capacities of C4-2 cells significantly decreased and the number of apoptotic cells increased. Accordingly, inhibiting SALL4 may suppress cell proliferation by facilitating apoptosis. Nevertheless, the mechanism by which this occurs is still unclear.

Cell apoptosis, as a multi-step procedure, is synergistically regulated by molecules and genes of various functions (Eum and Lee, 2011). Bcl-2 is a well-known anti-apoptotic gene and its overexpression decreases its downstream product Bax, which inhibits cell apoptosis and leads to disequilibrium between enhanced proliferation and weakened programmed cell death (Akl et al., 2014; Czabotar et al., 2014). Highly expressed in progressive and metastatic prostate cancers, Bcl-2 enhances the anti-apoptotic ability and eventually increases the tumor drug resistance of cancer cells (Furuya et al., 1996; Chan and Yu, 2004; Karnak and Xu, 2010). In this study, transient transfection of SALL4 siRNA promoted cell apoptosis, decreased Bcl-2 protein expression level and elevated that of Bax, suggesting that SALL4 was conducive to the apoptosis of prostate cancer cells. This is most likely mediated by the regulation of the expression of apoptosis-related proteins Bcl-2 and Bax. 
In summary, SALL4 is able to regulate several biological functions of prostate cancer cells. Downregulating SALL4 suppressed cell proliferation and colony formation as well as enhanced apoptosis most likely through Bcl-2 and Bax proteins. These findings pave the way for clarifying the mechanism by which SALL4 regulates cancer cells and its role in prostate cancer. However, this is only an in vitro study, so further experiments using animal models are still necessary.

\section{Conflicts of interest}

The authors declare no conflict of interest.

\section{ACKNOWLEDGMENTS}

We thank all the authors for their great contributions to this study.

\section{REFERENCES}

Akl H, Vervloessem T, Kiviluoto S, Bittremieux M, et al. (2014). A dual role for the anti-apoptotic Bcl-2 protein in cancer: mitochondria versus endoplasmic reticulum. Biochim. Biophys. Acta 1843: 2240-2252. http://dx.doi.org/10.1016/j. bbamcr.2014.04.017

Brumbaugh J, Hou Z, Russell JD, Howden SE, et al. (2012). Phosphorylation regulates human OCT4. Proc. Natl. Acad. Sci. USA 109: 7162-7168. http://dx.doi.org/10.1073/pnas.1203874109

Cao D, Li J, Guo CC, Allan RW, et al. (2009). SALL4 is a novel diagnostic marker for testicular germ cell tumors. Am. J. Surg. Pathol. 33: 1065-1077.http://dx.doi.org/10.1097/PAS.0b013e3181a13eef

Chan SL and Yu VC (2004). Proteins of the bcl-2 family in apoptosis signalling: from mechanistic insights to therapeutic opportunities. Clin. Exp. Pharmacol. Physiol. 31: 119-128. http://dx.doi.org/10.1111/j.1440-1681.2004.03975.x

Chisholm KM, Wan Y, Li R, Montgomery KD, et al. (2012). Detection of long non-coding RNA in archival tissue: correlation with polycomb protein expression in primary and metastatic breast carcinoma. PLoS One 7: e47998. http://dx.doi.org/10.1371/journal.pone.0047998

Czabotar PE, Lessene G, Strasser A and Adams JM (2014). Control of apoptosis by the BCL-2 protein family: implications for physiology and therapy. Nat. Rev. Mol. Cell Biol. 15: 49-63.http://dx.doi.org/10.1038/nrm3722

Eum KH and Lee M (2011). Crosstalk between autophagy and apoptosis in the regulation of paclitaxel-induced cell death in v-Ha-ras-transformed fibroblasts. Mol. Cell. Biochem. 348: 61-68.http://dx.doi.org/10.1007/s11010-010-0638-8

Forghanifard MM, Moghbeli M, Raeisossadati R, Tavassoli A, et al. (2013). Role of SALL4 in the progression and metastasis of colorectal cancer. J. Biomed. Sci. 20: 6. http://dx.doi.org/10.1186/1423-0127-20-6

Furuya Y, Krajewski S, Epstein JI, Reed JC, et al. (1996). Expression of bcl-2 and the progression of human and rodent prostatic cancers. Clin. Cancer Res. 2: 389-398.

Jemal A, Bray F, Center MM, Ferlay J, et al. (2011). Global cancer statistics. CA Cancer J. Clin. 61: 69-90.http://dx.doi. org/10.3322/caac. 20107

Karnak D and Xu L (2010). Chemosensitization of prostate cancer by modulating Bcl-2 family proteins. Curr. Drug Targets 11: 699-707.http://dx.doi.org/10.2174/138945010791170888

Kobayashi D, Kuribayshi K, Tanaka M and Watanabe N (2011). SALL4 is essential for cancer cell proliferation and is overexpressed at early clinical stages in breast cancer. Int. J. Oncol. 38: 933-939.

Lai YM, Huang H, Ling YH, Zeng LX, et al. (2013). Expression of SAL-like 4 protein in human prostate cancer tissue/ cell lines and its relationship with clinical outcome. Chin. J. Pathophysiol. 29: 2186-2192.

Liu N, Liang W, Ma X, Li X, et al. (2013). Simultaneous and combined detection of multiple tumor biomarkers for prostate cancer in human serum by suspension array technology. Biosens. Bioelectron. 47: 92-98. http://dx.doi. org/10.1016/j.bios.2013.02.052

Oikawa T, Kamiya A, Zeniya M, Chikada H, et al. (2013). Sal-like protein 4 (SALL4), a stem cell biomarker in liver cancers. Hepatology 57: 1469-1483. http://dx.doi.org/10.1002/hep.26159

Rao S, Zhen S, Roumiantsev S, McDonald LT, et al. (2010). Differential roles of Sall4 isoforms in embryonic stem cell pluripotency. Mol. Cell. Biol. 30: 5364-5380. http://dx.doi.org/10.1128/MCB.00419-10 
Yang J, Chai L, Gao C, Fowles TC, et al. (2008). SALL4 is a key regulator of survival and apoptosis in human leukemic cells. Blood 112: 805-813. http://dx.doi.org/10.1182/blood-2007-11-126326

Yong KJ, Gao C, Lim JS, Yan B, et al. (2013). Oncofetal gene SALL4 in aggressive hepatocellular carcinoma. N. Engl. J. Med. 368: 2266-2276. http://dx.doi.org/10.1056/NEJMoa1300297 